\title{
Recidiva bioquímica em câncer de próstata: artigo de revisão
}

\author{
Biochemical recurrence in prostate cancer: review article
}

\author{
Roberto Porto Fonseca', Amândio Soares Fernandes Junior', Volney Soares Lima², Stella Sala Soares Lima ${ }^{3}$, Alexandre Fonseca de Castro ${ }^{4}$, \\ Henrique de Lins e Horta ${ }^{4}$, Bruno Favato Neto ${ }^{5}$
}

\section{Resumo}

O câncer de próstata é a segunda neoplasia mais freqüente em homens, e a utilização do PSA levou a um aumento no número de casos diagnosticados com doença clinicamente localizada. A recidiva bioquímica é a elevação dos níveis de PSA em pacientes submetidos a tratamento com finalidade curativa para a doença localizada, na ausência de sinais clínicos ou radiológicos de recidiva. Neste artigo revisa-se a recidiva bioquímica, ressaltando-se a sua definição, a história natural e a abordagem terapêutica dos pacientes a fim de individualizar a condução dos casos com base nas evidências disponíveis.

Palavras-chave: Câncer de próstata; Recidiva bioquímica; Antígeno prostático específico

${ }^{1}$ Hospital Felício Rocho e ONCOMED, Belo Horizonte (MG)

${ }^{2}$ Hospital Felício Rocho, IPSEMG e ONCOMED, Belo Horizonte (MG)

${ }^{3}$ Hospital Luxemburgo, Belo Horizonte (MG)

${ }^{4}$ Hospital São Francisco de Assis e ONCOMED, Belo Horizonte (MG)

${ }^{5}$ Hospital São Lucas, Montes Claros (MG) 


\section{INTRODUÇÃO}

O câncer de próstata é a segunda neoplasia mais freqüente em homens. No Brasil, são estimados, para o ano de 2005, 46.330 casos, com taxa de incidência de 51,12 casos para cada 100.000 habitantes ${ }^{1}$.

A ampla utilização do PSA no diagnóstico e na avaliação dos pacientes com neoplasia de próstata, a partir da década de 80 , trouxe como importante conseqüência o aumento significativo do número de casos diagnosticados com doença clinicamente localizada e, conseqüentemente, da utilização de modalidades de tratamento com intenção curativa. Como exemplo, a taxa de prostatectomia radical (PR) aumentou de 17,4/100.000 em 1988 para 54,6/100.000 em 1992.

Outro importante fato ligado à utilização do PSA nos pacientes com câncer de próstata é a identificação de um grupo de pacientes que, após serem submetidos a tratamento com finalidade curativa para doença localizada, apresentam elevação do PSA na ausência de sinais clínicos ou radiológicos de recidiva. Nos Estados Unidos, dos cerca de 200.000 pacientes diagnosticados com câncer de próstata anualmente, estima-se que dois terços sejam tratados com cirurgia ou radioterapia (RT) e que $40 \%$ irão apresentar recidiva após o tratamento local, representando cerca de 50.000 pacientes por ano com diagnóstico de recidiva bioquímica.

\section{OBJETIVO}

Revisar a recidiva bioquímica, ressaltando a sua definição, a história natural e a abordagem terapêutica dos pacientes a fim de individualizar a condução dos casos com base nas evidências disponíveis.

\section{MÉTODOS}

Utilizou-se a base de dados Pubmed on line, sendo levantados os artigos publicados no período de 1997 a 2005, e selecionados aqueles de maior importância em relação ao número de citaçôes, à importância do periódico que publicou o artigo e ao julgamento dos autores.

\section{RESULTADOS}

O risco relativo para a recidiva bioquímica após a prostatectomia radical depende de fatores préoperatórios e fatores patológicos. Análises multivariadas apontam como principais fatores prognósticos o valor do PSA pré-operatório, estádio $\mathrm{T}$ patológico e o score de Gleason. Quatro grupos independentes de investigadores desenvolveram modelos matemáticos e nomogramas para a predição do risco de recidiva bioquímica $^{2-8}$ (Quadro 1). Além dos fatores citados, marcadores moleculares também vêm sendo avaliados como fatores de risco para a recidiva bioquímica. Bauer et al. demonstraram que a mutação no p53 e superexpressão do bcl-2 são fatores prognósticos independentes ${ }^{8}$.

\section{DEFININDO RECIDIVA BIOQUÍMICA}

Após uma prostatectomia radical, levando-se em conta que todo o tecido prostático foi inteiramente removido, a meia-vida sérica do PSA é de 2,6 dias; assim, dentro de duas a quatro semanas é esperado que o nível do PSA esteja abaixo de $0,1 \mathrm{ng} / \mathrm{mL}$ sendo considerado "indetectável". Níveis indetectáveis após uma cirurgia não são sinônimo de cura, visto que cerca de $40 \%$ dos pacientes irão progredir durante o

Quadro 1. Fatores prognósticos de recidiva bioquímica

\begin{tabular}{|c|l|l|}
\hline Autores & \multicolumn{1}{|c|}{ Parâmetros de predição } & \multicolumn{1}{|c|}{ End point avaliado } \\
\hline \multirow{3}{*}{ Baver et al. } & $\begin{array}{l}\text { Etnia } \\
\text { PSA pré-operatório } \\
\text { Gleason score } \\
\text { Acometimento da cápsula }\end{array}$ & Risco relativo de recidiva \\
\hline \multirow{3}{*}{ D Amico et al. } & $\begin{array}{l}\text { PSA pré-operatório } \\
\text { Estadiamento patológico } \\
\text { Margem cirúrgica } \\
\text { Gleason score }\end{array}$ & Taxa de falência de PSA em 2 anos \\
\hline \multirow{3}{*}{ Kattan et al. } & $\begin{array}{l}\text { PSA pré-operatório } \\
\text { Gleason score } \\
\text { Estadiamento patológico }\end{array}$ & Taxa de falência de PSA em 7 anos \\
\hline \multirow{2}{*}{ Partin et al. } & PSA pré-operatório & Risco relativo de recidiva \\
& Gleason score & \\
\hline
\end{tabular}


seguimento. A recidiva bioquímica pode ser definida como níveis de PSA persistentemente detectáveis após a PR ou um aumento do PSA após um período de normalização. Não existe um cut point de consenso para o diagnóstico de recidiva bioquímica. Algumas das principais séries da literatura ${ }^{9-11}$ (Quadro 2) utilizam $0,2 \mathrm{ng} / \mathrm{mL}$ e $0,4 \mathrm{ng} / \mathrm{mL}$. Cerca de $53 \%$ dos pacientes que atingem valores de PSA iguais a $0,2 \mathrm{ng} / \mathrm{mL}$ irão desenvolver recorrência clínica ao longo do tempo, e quando se utiliza um valor de $0,4 \mathrm{ng} / \mathrm{mL}$ observa-se que, aproximadamente, $80 \%$ dos pacientes evoluirão para recidiva clínica. Ensaios de PSA ultra-sensível, apesar de não estarem incorporados ainda na prática clínica diária, talvez possam detectar a recidiva bioquímica alguns meses ou anos antes que os ensaios convencionais disponíveis ${ }^{12-14}$, porém ainda não se sabe sobre os reais benefícios de sua utilização.

Quadro 2. Valores de corte para diagnóstico de recidiva bioquímica após prostatectomia radical

\begin{tabular}{|l|c|}
\hline \multicolumn{1}{|c|}{ Autores } & Cut point \\
\hline $\begin{array}{l}\text { Johns Hopkins / } \\
\text { Mayo Clinic }\end{array}$ & $0,2 \mathrm{ng} / \mathrm{mL}$ \\
\hline Kattan et al. & $0,4 \mathrm{ng} / \mathrm{mL}$ \\
\hline Moul et al. & 2 valores acima de \\
$0,2 \mathrm{ng} / \mathrm{mL}$
\end{tabular}

A interpretação dos valores do PSA após a RT externa ainda é mais complexa visto que a próstata permanece in situ. Ocorre uma elevação do PSA na fase inicial do tratamento provocada pelo dano celular e necrose; após observa-se uma evolução bifásica caracterizada por uma queda rápida dos níveis de PSA e, a seguir, um declínio mais lento, porém gradual dos seus níveis séricos. A meia-vida do PSA após a radioterapia externa varia entre
1,9 e 3 meses e o tempo médio para o nadir do PSA após essa modalidade de tratamento é de 18 meses, podendo haver flutuaçōes. Segundo o consenso da American Society for Therapeutic Radiology and Oncology $(\mathrm{ASTRO})^{15}$, a recidiva bioquímica após a radioterapia pode ser definida como três aumentos consecutivos do PSA a partir do nadir após a radioterapia. O valor do nadir é um importante fator prognóstico, porém não existe um valor que determine o sucesso ou o insucesso do tratamento. Modificações nos critérios de definição de recidiva bioquímica pós-radioterapia foram propostas recentemente ${ }^{9}$, já que teriam uma maior sensibilidade e especificidade para predizer recidiva clínica (local ou à distância) do que os da ASTRO.

\section{HISTÓRIA NATURAL DA RECIDIVA BIOQUÍMICA}

Os pacientes com diagnóstico de recidiva bioquímica constituem um grupo bastante heterogêneo em relação ao desenvolvimento de doença metastática. No estudo da história natural dos pacientes com recidiva bioquímica após prostatectomia radical, merece destaque o trabalho de Pound et al. publicado em $1999^{16}$ e atualizado na ASCO de $2003^{17}$. Nesse estudo, foram acompanhados 329 pacientes com diagnóstico de recidiva bioquímica, de um total de 3.263 pacientes submetidos à PR entre 1982 e 2002 . Ressalta-se que os pacientes não fizeram uso de hormonioterapia até o diagnóstico de doença metastática. A mediana do tempo para o desenvolvimento de doença metastática foi de 7,5 anos e a mediana do tempo entre o diagnóstico de metástase e o óbito foi de 6,5 anos. Os principais fatores preditores da probabilidade e do tempo para o desenvolvimento de doença metastática foram o score de Gleason (5-7 x 8-10), o tempo para o diagnóstico de recidiva bioquímica ( $>2$ anos $\mathrm{x}<2$ anos) e o tempo de dobramento do PSA $(>10$ meses $x<10$ meses $)$, conforme apresentado na Tabela 1 .

Tabela 1. Probabilidade de sobrevida livre de metástases após recidiva bioquímica, segundo Pounds et al.

\begin{tabular}{|c|c|c|c|c|c|c|c|c|}
\hline \multirow{3}{*}{$\begin{array}{c}\text { Gleason } \\
\text { Ano da } \\
\text { recorrência } \\
\text { após a PR } \\
\text { Tempo de } \\
\text { dobramento do } \\
\text { PSA }\end{array}$} & \multicolumn{4}{|c|}{ 5-7 anos } & \multicolumn{4}{|c|}{ 8-10 anos } \\
\hline & \multicolumn{2}{|c|}{$>2$ anos } & \multicolumn{2}{|c|}{$<2$ anos } & \multicolumn{2}{|c|}{$>2$ anos } & \multicolumn{2}{|c|}{$<2$ anos } \\
\hline & $\begin{array}{c}>10 \\
\text { meses }\end{array}$ & $\begin{array}{c}<10 \\
\text { meses }\end{array}$ & $\begin{array}{c}>10 \\
\text { meses }\end{array}$ & $\begin{array}{c}<10 \\
\text { meses }\end{array}$ & $\begin{array}{c}>10 \\
\text { meses }\end{array}$ & $\begin{array}{c}<10 \\
\text { meses }\end{array}$ & $\begin{array}{c}>10 \\
\text { meses }\end{array}$ & $\begin{array}{c}<10 \\
\text { meses }\end{array}$ \\
\hline 3 anos & $95 \%$ & $68 \%$ & $99 \%$ & 77\% & $95 \%$ & $62 \%$ & NA & $54 \%$ \\
\hline 5 anos & $92 \%$ & $40 \%$ & $95 \%$ & $42 \%$ & $73 \%$ & $43 \%$ & NA & $30 \%$ \\
\hline 7 anos & $87 \%$ & $34 \%$ & $84 \%$ & $24 \%$ & $60 \%$ & $26 \%$ & NA & $21 \%$ \\
\hline
\end{tabular}


Outro estudo publicado por Freedland et al. ${ }^{18}$ analisou retrospectivamente 379 pacientes submetidos à prostatectomia radical por um tempo de seguimento mediano de 10 anos, e estratificou o risco de mortalidade por câncer de próstata pelos mesmos parâmetros que o trabalho de Pound et al., porém com cutoffs diferentes para o tempo de dobramento do PSA $(<3,0$ meses, 3,08,9 meses, 9,0-14,9 meses, $>15$ meses) e para o tempo até o diagnóstico de recidiva bioquímica ( $=3$ anos versus $>3$ anos). A estimativa para a sobrevida em 15 anos relacionada ao câncer de próstata de acordo com este trabalho é apresentada na Tabela 2.

De um modo semelhante, Zelefsky et al. ${ }^{19}$ avaliaram a história natural dos pacientes com recidiva bioquímica após radioterapia externa, em um estudo recentemente publicado. Nesse estudo, os principais fatores preditores do risco de doença metastática foram o estádio clínico, o score de Gleason e o tempo de dobramento do PSA (Tabela 3).

Recentemente Zhou et al. ${ }^{20}$ também mostraram que o risco de morte por câncer de próstata após a recidiva bioquímica (tanto pós-prostatectomia quanto pósradioterapia) está relacionado ao tempo de dobramento do PSA e ao Gleason score.

\section{AvaliaçaO e tratamento}

O objetivo da avaliação inicial do paciente com recidiva bioquímica é determinar se esta traduz uma recorrência local ou sistêmica do câncer de próstata, o que é justificado pela existência de diferentes modalidades terapêuticas em cada uma dessas situaçôes.

Os dados indicam que a maior parte das recidivas bioquímicas é manifestação de doença metastática. Pound et al. ${ }^{16}$ reportaram que, após a recidiva bioquímica, aproximadamente um terço dos pacientes apresentam doença localizada e dois terços doença metastática, acompanhada ou não de recorrência local. Outros investigadores também relatam uma baixa incidência de recidiva local isolada, variando de $10 \%$ a $25 \%$.
A determinação precisa do sítio de recidiva é difícil, sendo muitas vezes impossível. O toque retal, a cintilografia óssea, a tomografia computadorizada, o prostascint, a ressonância nuclear magnética e a biopsia prostática transretal possuem baixa sensibilidade e especificidade. A cintilografia óssea, por exemplo, foi avaliada por Cher e Bianco em 93 pacientes com recidiva bioquímica após $\mathrm{PR}$, e o menor valor do PSA associado à cintilografia óssea anormal foi de $46 \mathrm{ng} / \mathrm{m}^{21}$.

Em virtude do valor limitado dos exames de imagem na avaliação dos pacientes com recidiva bioquímica, vários investigadores reforçam a importância de fatores histológicos bem como os ligados à cinética do PSA para a distinção entre uma recidiva local e uma sistêmica. Sabe-se que em um paciente com score de Gleason maior do que 7 , com recidiva bioquímica que ocorre em menos de dois anos do tratamento definitivo e tempo de dobramento do PSA menor do que seis meses, a probabilidade de recorrência local exclusiva é muito baixa.

Não existe uma conduta-padrão para os pacientes com recidiva bioquímica. As modalidades terapêuticas mais freqüentemente adotadas são observação, radioterapia de salvamento e hormonioterapia. Em casos selecionados também se pode utilizar a braquiterapia, a crioterapia e a prostatectomia de salvamento.

A radioterapia de resgate, após tratamento inicial com PR, é tratamento a ser considerado em pacientes

Tabela 3. Risco de metástases de câncer de próstata em 3 anos, de acordo com o tempo de dobramento do PSA na recidiva bioquímica

\begin{tabular}{c|c}
\hline PSADT & $\begin{array}{c}\text { Doença metastática } \\
\text { em } 3 \text { anos }\end{array}$ \\
\hline $0-3$ meses & $49 \%$ \\
\hline $3-6$ meses & $41 \%$ \\
\hline $6-12$ meses & $20 \%$ \\
\hline$>12$ meses & $7 \%$ \\
\hline
\end{tabular}

Tabela 2. Estimativa de sobrevida em 15 anos relacionada ao câncer de próstata em pacientes com recidiva bioquímica tratados com prostatectomia radical, segundo Freedland et al.

\begin{tabular}{|c|c|c|c|c|}
\hline \multirow{3}{*}{$\begin{array}{c}\text { Tempo de dobramento } \\
\text { do PSA }\end{array}$} & \multicolumn{4}{|c|}{ Estimativa de sobrevida em 15 anos (\%) } \\
\hline & \multicolumn{2}{|c|}{$\begin{array}{c}\text { Recorrência }>3 \text { anos após } \\
\text { prostatectomia }\end{array}$} & \multicolumn{2}{|c|}{$\begin{array}{c}\text { Recorrência } \leq 3 \text { anos após } \\
\text { prostatectomia }\end{array}$} \\
\hline & $\begin{array}{c}\text { Gleason score } \\
<8\end{array}$ & $\begin{array}{l}\text { Gleason score } \\
\geq 8\end{array}$ & $\begin{array}{c}\text { Gleason score } \\
<8\end{array}$ & $\begin{array}{c}\text { Gleason score } \\
\geq 8\end{array}$ \\
\hline$\geq 15$ meses & $94(87$ a 100$)$ & 87 (79 a 92) & 81 (57 a 93) & 62 (32 a 85) \\
\hline $9,0-14,9$ meses & $86(57$ a 97$)$ & 72 (35 a 92) & $59(24$ a 87$)$ & $31(7$ a 72$)$ \\
\hline $3,0-8,9$ meses & 59 (32 a 81) & $30(10$ a 63$)$ & 16 (4 a 49) & $1(<1$ a 51$)$ \\
\hline$<3$ meses & $19(5$ a 51$)$ & $2(<1$ a 38$)$ & $<1(<1$ a 26$)$ & $<1(<1$ a 2$)$ \\
\hline
\end{tabular}


em que se sugere que a recorrência seja local. Estudos relatam taxa de sobrevida livre de recidiva bioquímica de $18 \%$ a $68 \%$, sendo a maioria dos pacientes em estádio cirúrgico T3, níveis de PSA da recidiva menores que $2 \mathrm{ng} / \mathrm{mL}$, mas com tempo de seguimento curto após a RT de resgate. Os principais fatores que influenciam o sucesso da RT de resgate são os mesmos relacionados ao risco de doença metastática (estádio inicial, score de Gleason, tempo de recidiva após tratamento definitivo e tempo de dobramento do PSA). Segundo consenso da ASTRO, quando indicada RT de resgate, recomenda-se que seja iniciada antes que o nível de PSA atinja $1,5 \mathrm{ng} / \mathrm{mL}$ e que se utilizem doses maiores que 64Gy. Outros autores sugerem que a radioterapia adjuvante seja iniciada assim que ocorra elevação do PSA após o tratamento cirúrgico, principalmente em pacientes com margens cirúrgicas comprometidas ou estádio patológico T3.

O melhor manejo do paciente com recidiva bioquímica após RT externa permanece controverso. A prostatectomia de resgate após RT não era amplamente considerada devido a elevadas taxas de complicações per e pós-operatórias (40\%-50\%), com altas taxas de recidivas. Entre as principais complicações destaca-se lesão retal (6-15\%), estenose de colo de bexiga (20-28\%) e incontinência urinária (40\%-50\%). Entretanto, estudos mais recentes, em grupo de pacientes selecionados (PSA pré-RT $<10 \mathrm{ng} / \mathrm{mL}$, PSA após RT $<4 \mathrm{ng} / \mathrm{mL}$, score de Gleason $<6$, e estádio T1c ou T2a), descrevem melhores taxas de sobrevida livre de recidiva, embora ainda com elevada morbidade. Não existem dados suficientes para avaliar os resultados da $\mathrm{PR}$ de resgate em pacientes submetidos inicialmente à braquiterapia, destacandose a morbidade ainda mais elevada dessa associação.

Em relação ao emprego da ablação hormonal, não existe nenhum estudo prospectivo randomizado em pacientes com recidiva bioquímica, portanto seu real valor não pode ser determinado até o presente momento. Os trabalhos mais citados pelos defensores da hormonioterapia precoce nos pacientes com recidiva bioquímica incluem pacientes com doença localmente avançada e doença metastática.

No estudo EORTC 22863, Bolla et al. ${ }^{22}$. randomizaram 401 pacientes com adenocarcinoma de próstata localmente avançado para radioterapia isolada ou associada à ablação hormonal, por três anos. Após cinco anos de seguimento, observou-se um aumento na sobrevida estatisticamente significativo, beneficiando o grupo tratado com ablação hormonal (79\% x 62\%).

Messing et al., no estudo ECOG 788723, avaliaram uma população de 98 pacientes submetidos à prostatectomia radical e linfadenectomia que apresentavam comprometimento linfonodal. Os pacientes foram randomizados para receber hormonioterapia precocemente ou serem observados até a progressão. Após 10 anos de seguimento, os pacientes tratados precocemente tiveram uma maior sobrevida global $(72,4 \% \times 49 \%$; $=0,025)$ bem como uma maior sobrevida causa específica (82,20\% x 56,9\%; p=0,001).

Em um outro importante estudo, o Medical Research Council ${ }^{4}$ avaliou 938 pacientes com câncer de próstata localmente avançado e metastático, demonstrando que a utilização de hormonioterapia precoce reduz de maneira significativa a morbidade relacionada ao câncer de próstata, bem como aumenta a sobrevida causa específica.

A extrapolação dos resultados desses estudos para os pacientes com recidiva bioquímica está longe do ideal. Acresça-se que os efeitos indesejados da hormonioterapia não devem ser subestimados, particularmente em um grupo de pacientes sem sintomas clínicos da doença.

Assim, o manejo dos pacientes com recorrência bioquímica do câncer de próstata é um desafio para oncologistas, urologistas e radioterapeutas. Trata-se, sem dúvida, de um grupo de pacientes com evolução clínica variada, cujo conhecimento dos fatores prognósticos é de fundamental importância, pois permite poupar exames onerosos e muitas vezes desnecessários, e individualizar o tratamento da melhor forma possível.

\section{REFERÊNCIAS}

1. Jemal A, Tiwari RC, Murray T, et al. Cancer statistics, 2004. CA Cancer J Clin. 2004;54:8-29.

2. D'Amico AV, Cote K, Loffredo M, et al. Determinants of prostate cancer-specific survival after radiation therapy for patients with clinically localized prostate cancer. J Clin Oncol. 2002;20:4567-573.

3. D'Amico AV, Moul JW, Carroll PR, et al. Surrogate end point for prostate cancer-specific mortality after radical prostatectomy or radiation therapy. J Natl Cancer Inst. 2003;95:1376-383.

4. D'Amico AV, Moul JW, Carroll PR, et al. Intermediate end point for prostate cancer-specific mortality following salvage hormonal therapy for prostate-specific antigen failure. J Natl Cancer Inst. 2004;96:509-15.

5. Partin AW, Eisenberger MA, Sinibaldi VJ, et al. Prostate specific antigen doubling time (PSADT) predicts for distant failure and prostate cancer specific survival (PCSS) in men with biochemical relapse after radical prostatectomy (RP) [Abstract no 4555]. Proc Am Soc Clin Oncol. 2004;23:394a.

6. Kattan MW, Zelefsky MJ, Kupelian PA, Scardino PT, Fuks Z, Leibel SA. Pretreatment nomogram for predicting the outcome of three-dimensional conformal radiotherapy in prostate cancer. J Clin Oncol. 2000;18(19):3352-359. 
7. Kattan MW, Potters L, Blasko JC, Beyer DC, Fearn P, Cavanagh W, et al. Pretreatment nomogram for predicting freedom from recurrence after permanent prostate brachytherapy in prostate cancer. Urology. 2001;58(3):393-99.

8. Bauer JJ, Srivastava S, Connelly RR, Sesterhenn IA, Preston DM, McLeod DG, et al. Significance of familial history of prostate cancer to traditional prognostic variables, genetic biomarkers, and recurrence after radical prostatectomy. Urology. 1998;51(6):970-76.

9. Horwitz EM, Thames HD, Kuban DA, et al. Definitions of biochemical failure that best predict clinical failure in patients with prostate cancer treated with external beam radiation alone: a multi-institutional pooled analysis. J Urol. 2005;173:797-802.

10. Zhou P, Chen MH, McLeod D, Carroll PR, Moul JW, D'Amico AV. Predictors of prostate cancer-specific mortality after radical prostatectomy or radiation therapy. J Clin Oncol. 2005;23(28):6992-998

11. Amling CL, Bergstralh EJ, Blute ML, Slezak JM. Defining prostate specific antigen progression after radical prostatectomy: what is the most appropriate cut point? J Urol. 2001;165:1146-151.

12. Haese A, Huland E, Graefen M, Hammerer P. Ultrasensitive detection of prostate specific antigen in the follow-up of 422 patients after radical prostatectomy. J Urol. 1999;161:1206-211.

13. Shen S, Lepor H, Yaffee R, Taneja SS. Ultrasensitive serum prostate specific antigen nadir accurately predicts the risk of early relapse after radical prostatectomy. J Urol. 2005;173:777-80.

14. Witherspoon LR, Lapeyrolerie T. Sensitive prostate specific antigen measurements identify men with long disease-free intervals and differentiate aggressive from indolent cancer recurrences within 2 years after radical prostatectomy. J Urol. 1997; 157:1322-328.

15. American Society for Therapeutic Radiology and Oncology. Consensus statement: guidelines for PSA following radiation therapy. American Society for Therapeutic Radiology and Oncology Consensus Panel. Int J Radiat
Oncol Biol Phys. 1997;37:1035-1041.

16. Pound CR, Partin AW, Eisenberger MA, et al. Natural history of progression after PSA elevation following radical prostatectomy. JAMA. 1999;281:1591-597.

17. Eisenberger ME, Partin AW, Pound C, et al. Natural history of progression of patients with biochemical (PSA) relapse following radical prostatectomy: Update [Abstract $n^{\circ}$ 1527]. Proc Am Soc Clin Oncol. 2003;21:380a.

18. Freedland SJ, Humphreys EB, Mangold LA, et al. Risk of prostate cancer-specific mortality following biochemical recurrence after radical prostatectomy. JAMA. 2005;294:433-39.

19. Zelefsky MJ, Scher HI, Cham MH, et al. Outcome predictors for the increasing PSA state after definitive external-beam radiotherapy for prostate cancer. J Clin Oncol. 2005:23:826-31.

20. Zhou P, Chen MH, McLeod D, Carroll PR, Moul JW, D'Amico AV. Predictors of prostate cancer-specific mortality after radical prostatectomy or radiation therapy. J Clin Oncol. 2005;23(28):6992-998

21. Cher ML, Bianco FJ, Lam JS, et al. Limited role of radionuclide bone scintigraphy in patients with prostate antigen elevations after radical prostatectomy. J Urol. 1998;160:1387-391.

22. Bolla M, Collette L, Blank L, et al. Long-term results with immediate androgen suppression and external irradiation in patients with locally advanced prostate cancer (an EORTC study): a phase III randomised trial. Lancet. 2002;360:103-106.

23. Messing EM, Manola J, Sarosdy M, et al. Immediate hormonal therapy versus observation after radical prostatectomy and pelvic lymphadenectomy for node positive prostate cancer: At 10 years results of EST3886 [Abstract]. Proc Am Soc Clin Oncol. 2004;23:398a.

24. The Medical Research Council Prostate Cancer Working Party Investigators Group. Immediate versus deferred treatment for advanced prostatic cancer: initial results of the Medical Research Council Trial. Br J Urol. 1997;79:235-46.

\begin{abstract}
Prostate cancer is the second most common neoplasm in men, and the routine use of PSA has led to an increase in the diagnosis of clinically localized disease. Biochemical recurrence is defined as a rise in PSA levels without overt clinical or radiological disease in patients who have received treatment with the intent to cure localized disease, in the absence of clinical or radiological signs of recurrence. In this article, we review biochemical recurrence with an emphasis on its definition, natural history, and treatment, in order to individualize case management based on current evidence.
\end{abstract}

Key words: Prostate cancer; Biochemical recurrence; Prostate specific antigen 